\title{
ARTICLE
}

\section{The structure of Zika virus NS5 reveals a conserved domain conformation}

Boxiao Wang ${ }^{1, \star}$, Xiao-Feng Tan ${ }^{1, \star}$, Stephanie Thurmond ${ }^{2}$, Zhi-Min Zhang ${ }^{1}$, Asher Lin ${ }^{1}$, Rong Hai ${ }^{2} \&$ Jikui Song ${ }^{1}$

The recent outbreak of Zika virus (ZIKV) has imposed a serious threat to public health. Here we report the crystal structure of the ZIKV NS5 protein in complex with $S$-adenosyl-L-homocysteine, in which the tandem methyltransferase (MTase) and RNA-dependent RNA polymerase (RdRp) domains stack into one of the two alternative conformations of flavivirus NS5 proteins. The activity of this NS5 protein is verified through a de novo RdRp assay on a subgenomic ZIKV RNA template. Importantly, our structural analysis leads to the identification of a potential drug-binding site of ZIKV NS5, which might facilitate the development of novel antivirals for ZIKV.

\footnotetext{
${ }^{1}$ Department of Biochemistry, University of California, Riverside, Riverside, California 92521, USA. ${ }^{2}$ Department of Plant Pathology and Microbiology, University of California, Riverside, Riverside, California 92521, USA. * These authors contributed equally to this work. Correspondence and requests for materials should be addressed to R.H. (email: ronghai@ucr.edu) or to J.S. (email: jikui.song@ucr.edu).
} 
T he outbreak of Zika virus (ZIKV) in the Americas and West Pacific islands in the past year has become a worldwide health concern, affecting more than 60 countries to date ${ }^{1,2}$. Increasing evidence has linked ZIKV infection to microcephaly in newborn infants ${ }^{3}$, and to Guillain-Barré syndrome in adults ${ }^{4}$. The fact that no vaccines or therapeutics for prevention or treatment of ZIKV infection are currently available further deepens the concern. To develop effective antivirals against ZIKV infection, it is urgent to gain a comprehensive structural and mechanistic understanding of the molecular machineries underpinning the life cycle of ZIKV.

ZIKV belongs to the family of flavivirus that includes a variety of mosquito-borne human pathogens, such as dengue virus (DENV1-4), yellow fever virus, West Nile virus, Spondweni virus and Japanese encephalitis virus (JEV) ${ }^{5}$. The genome of ZIKV is organized into the form of a single positive strand RNA, encoding in total three structural proteins $(\mathrm{C}, \mathrm{prM} / \mathrm{M}$ and $\mathrm{E})$ and seven non-structural (NS) proteins (NS1, NS2A, NS2B, NS3, NS4A, NS4B and NS5) ${ }^{6}$. Among these, NS5 is the largest NS protein, containing an $\mathrm{N}$-terminal methyltransferase (MTase) domain responsible for viral RNA capping and a C-terminal RNA-dependent RNA polymerase (RdRp) domain for viral RNA synthesis, with evidence indicating that the MTase and RdRp domains cooperate in RNA synthesis initiation and elongation ${ }^{7}$. In addition, NS5 proteins have been shown to inhibit the type I interferon (IFN) signalling to evade antiviral defence in the host ${ }^{8-16}$. The essential role of NS5 in viral replication and immunosuppression makes it an ideal target for antivirals ${ }^{17}$. However, the molecular mechanism underlying the enzymatic action of NS5 remains poorly understood. Thus far, crystal structures of full-length NS5 proteins have only been reported for JEV and DENV3 (refs 18,19). Intriguingly, despite with $\sim 65 \%$ sequence identity (Supplementary Fig. 1), JEV NS5 and DENV3 NS5 adopt different orientations between the MTase and RdRp domains ${ }^{18,19}$, raising the question of how the MTase and RdRp domains of NS5 cooperate during RNA replication and capping.
To illuminate the structure and mechanism of NS5 proteins, and, more importantly, to explore potential druggable sites for ZIKV, we determined the crystal structure of full-length ZIKV NS5 in complex with S-adenosyl-L-homocysteine (SAH), by-product of cofactor $S$-adenosyl-L-methionine at $3.3 \AA$ resolution. Our structural analysis reveals that ZIKV NS5 folds into one of the two alternative conformations of flavivirus NS5 proteins, providing functional implication for the conformational dynamics of flavivirus NS5 proteins. We further verify the enzymatic activity of ZIKV NS5 through a de novo RdRp assay using a subgenomic ZIKV RNA as template. Finally, we show that the structure of ZIKV NS5 provides a framework for future development of novel antivirals against ZIKV infection.

\section{Results}

Overall structure of ZIKV NS5. We were able to trace nearly the entire sequence of ZIKV NS5 (Fig. 1a), except for the first five $\mathrm{N}$-terminal residues, residues 747-748 in the RdRp domain, and sixteen residues at the $\mathrm{C}$ terminus. The structure reveals an $\mathrm{N}$-terminal classic $S$-adenosyl-L-methionine-dependent MTase domain situated on top of a C-terminal RdRp domain. The MTase domain is dominated by a Rossmann fold, with a sevenstranded $\beta$-sheet sandwiched by two $\alpha$-helices from one side and another $\alpha$-helix from the other side. Near to the catalytic site, the SAH molecule is surrounded by a set of conserved residues (Supplementary Fig. 2). In addition, the N-terminal extension sequence $(\alpha 1-\alpha 4$ in Supplementary Fig. 1$)$ of the MTase domain pairs with its C-terminal extension sequence $(\alpha 8-\beta 9$ in Supplementary Fig. 1) to form a helix bundle and an antiparallel two-stranded $\beta$-sheet, adding another structural layer onto the Rossmann fold (Fig. 1b). As with other viral RdRps ${ }^{20}$, the ZIKV NS5 RdRp adopts a capped right-hand structure with the Palm, Fingers and Thumb subdomains and a priming sequence poised to receive RNA substrates (Fig. 1b). The RdRp domain also harbours two zinc ions, as observed for the NS5 proteins of JEV

a

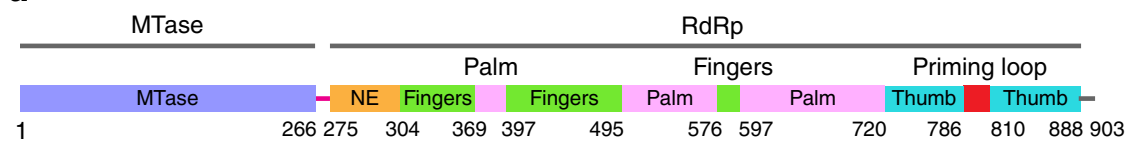

b
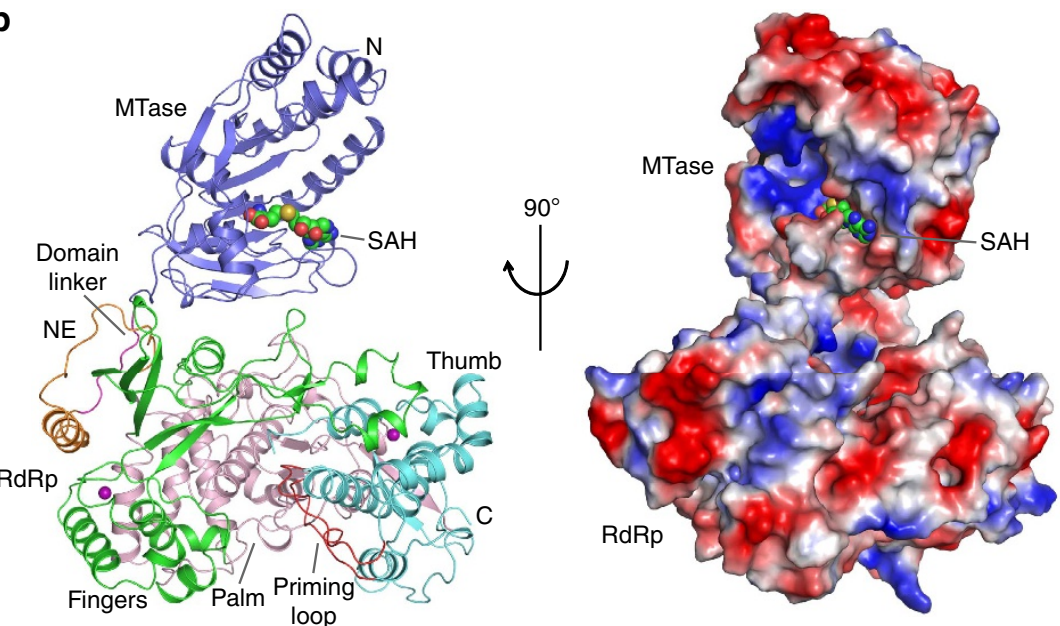

Figure 1 | Structural overview of ZIKV NS5. (a) Colour-coded domain architecture of ZIKV NS5. (b) Orthogonal views of ribbon (left) and electrostatic surface (right) representations of ZIKV NS5. The MTase domain, the N-terminal extension, palm, fingers, priming loop and thumb of the RdRp domain, and the interdomain linker are coloured in slate, orange, pink, green, red, light blue and magenta, respectively. Zinc ions (purple) and $\mathrm{SAH}$ are shown in sphere representation. 
a

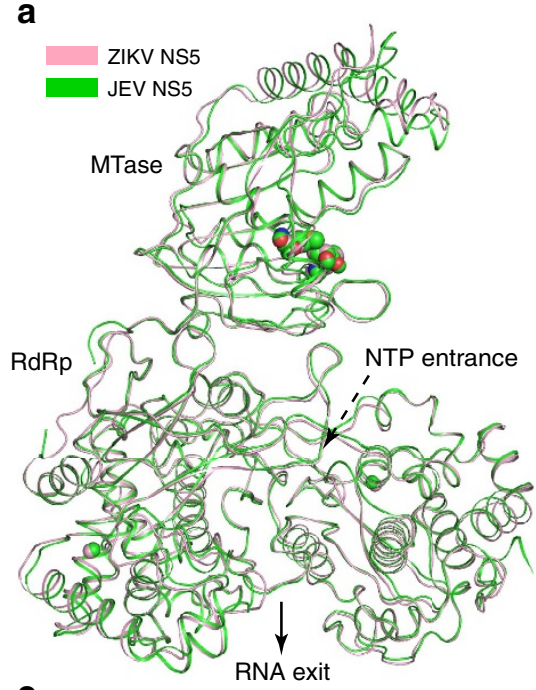

c

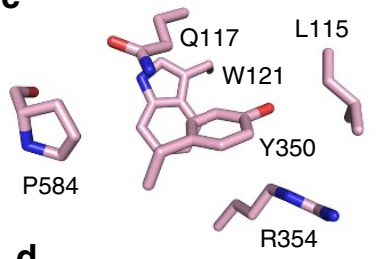

d $\quad$ Y119

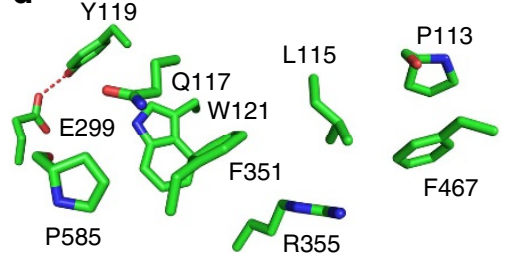

b

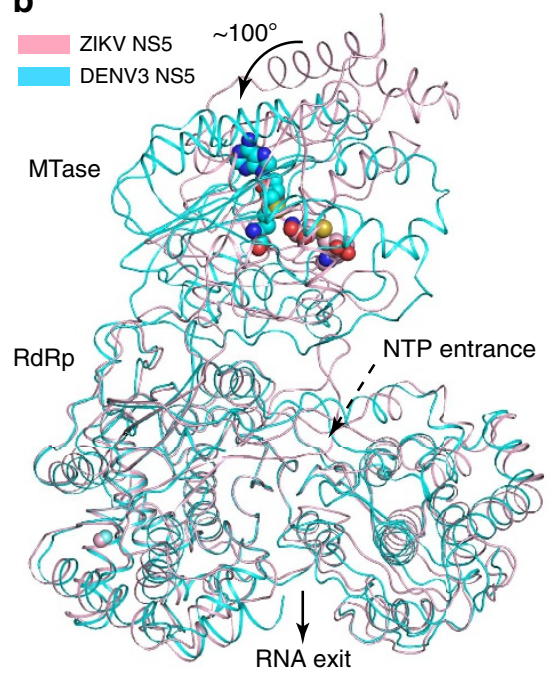

e

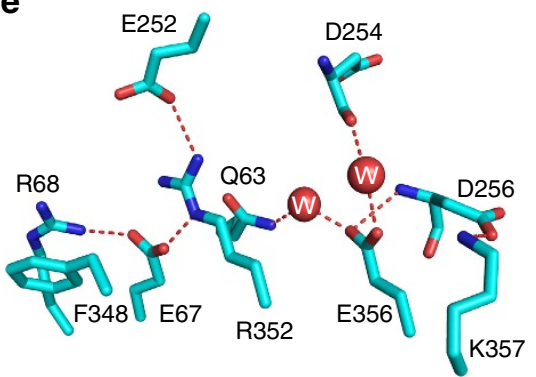

Figure 2 | Structural comparison of NS5 proteins from ZIKV and two other flaviviruses. Structural superposition of ZIKV NS5 with (a) JEV NS5 (PDB $4 \mathrm{~K} 6 \mathrm{M}$ ) and (b) DENV3 NS5 (PDB 4VOQ). Alignment of the RdRp domains of ZIKV NS5 and DENV3 NS5 leads to a $\sim 100^{\circ}$ change in orientation between the MTase and RdRp domains. The NTP entrance and RNA exit sites are labelled. (c-e) The MTase-RdRp domain interactions of (c) ZIKV NS5, (d) JEV NS5 and (e) DENV3 NS5.

and DENV3 (refs 18,19).The associations of the MTase domain with the RdRp domain does not involve extensive interdomain contacts, leading to a modest buried surface area of $\sim 1,400 \AA^{2}$. In fact, structural superposition of the MTase domain in full-length NS5 and the recently reported domain alone ${ }^{21}$ gives a root-mean-square deviation (RMSD) of $0.42 \AA$ over $242 \mathrm{C} \alpha$ atoms, indicating that the MTase-RdRp association does not lead to considerable conformational change of the MTase domain.

Structural comparison with other flavivirus NS5 proteins. ZIKV NS5 shares $\sim 68 \%$ and $\sim 66 \%$ sequence identity, respectively, with its JEV and DENV3 counterparts (Supplementary Fig. 1). However, these NS5 homologues appear to antagonize the IFN signalling through different mechanisms: JEV NS5 suppresses IFN signalling likely through blocking phosphorylation of the IFN signalling components ${ }^{13}$, whereas DENV3 NS5 and ZIKV NS5 inhibit the IFN signalling through promoting protein degradation of signal transducer and activator of transcription 2 in an E3 ubiquitin ligase UBR4-dependent or -independent manner ${ }^{10,16}$. Along the line, we compared the structure of ZIKV NS5 with those of JEV NS5 and DENV3 NS5 (Fig. 2a,b). Remarkably, ZIKV NS5 superimposes well with JEV NS5, with an RMSD of $0.63 \AA$ over $872 \mathrm{C} \alpha$ atoms (Fig. 2a). In particular, the MTase-RdRp associations of ZIKV NS5 and JEV NS5 are both mediated by the same set of van de Waals contacts, involving the
C-terminal extension of the MTase domain (P113, L115, Q117 and W121), and the Index, Ring and Middle fingers of the RdRp domain (Y350, R354, F466 and P584 in ZIKV NS5) (Fig. 2c,d). Subtle structural divergence between ZIKV NS5 and JEV NS5 was mainly observed for the $\mathrm{N}$ - and C-terminal extension of the MTase domain, the MTase-RdRp domain linker, and a segment in the Palm subdomain (residues E632-G653 in ZIKV NS5) (Supplementary Fig. 3a). Note that these regions have previously been linked to NS5-mediated immunosuppression ${ }^{13,14}$. Therefore, such structural divergence may underlie the distinct mechanisms of ZIKV NS5 and JEV NS5 in IFN antagonism. By contrast, structural superposition of ZIKV NS5 and DENV3 NS5 gives a RMSD of $6.06 \AA$ over $844 \mathrm{C} \alpha$ atoms, attributed in large part to the difference in the relative orientation between the MTase and RdRp domains (Fig. 2b). Unlike the structure of ZIKV NS5 in which the MTase domain sits on the back of the RdRp domain, the MTase domain of DENV3 NS5 approaches towards the front of the RdRp domain, resulting in a more compact conformation (Fig. 2b). Distinct from those of ZIKV NS5 (Fig. 2c,d) and JEV NS5, the MTase-RdRp association of DENV3 is mediated by a set of hydrogen bonding, cation- $\pi$ and electrostatic interactions between the N- (Q63, E67 and R68) and C-terminal extensions (E252 and D254) of the MTase domain and the Index finger (F348, R352, E356 and K357) of the RdRp domain (Fig. 2e). These interactions of DENV3 NS5 appear to draw the MTase domain towards the NTP entrance of the RdRp domain, resulting in a $\sim 100^{\circ}$ rotation of the MTase 
a
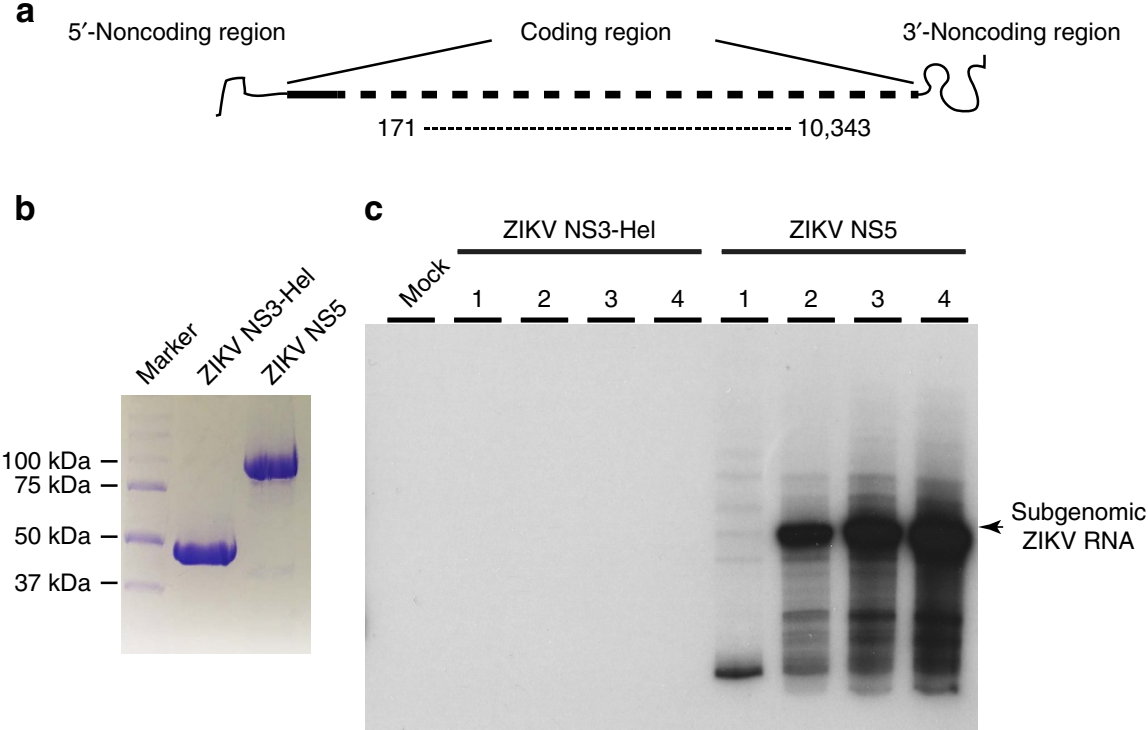

Figure 3 | De novo RNA synthesis by ZIKV NS5 protein. (a) The subgenomic ZIKV RNA contains an internal deletion from nucleotides 171 to 10,343 (GenBank accession no. KU963573.2). (b) SDS-polyacrylamide gel electrophoresis analysis of purified ZIKV NS5 and ZIKV NS3-Hel. (c) ZIKV de novo RNA replication assay. The subgenomic ZIKV RNA was incubated with recombinant ZIKV NS5 protein, ZIKV NS3-Hel or alone (mock). The relative amount of ${ }^{32}$ P-labelled RNA product is displayed in the autoradiograph of the PAGE gel. The reactions containing recombinant proteins were divided into four groups. Group1 was incubated at $23^{\circ} \mathrm{C}$ for $30 \mathrm{~min}$. Groups 2, 3 and 4 were incubated at $33^{\circ} \mathrm{C}$ for 30,60 or 120 min, respectively (Supplementary Fig. 6 ).

domain in relation to the $\mathrm{RdRp}$ domain (Fig. 2b). Another prominent structural difference between ZIKV/JEV NS5 and DENV3 NS5 arises from the substrate binding motifs of RdRp, including motif $F$ in the Ring finger and motif $G$ in the Pinky finger ${ }^{22,23}$ (Fig. 2b). The conformations of these two motifs appear to be stabilized by the MTase-Ring finger association of ZIKV/JEV NS5, but become disordered in DENV3 NS5 due to the loss of the corresponding interactions (Supplementary Fig. 3b).

The fact that the structure of ZIKV NS5 exhibits an extended domain conformation similar to that of JEV NS5, but differently from that of DENV3 NS5, raises a question on the functional implication of these two conformational states of NS5 proteins. On one hand, it is likely that the structures of ZIKV/JEV NS5 diverge from that of DENV3 NS5 through adaptive mutations of specific regions (for example, domain linker) during evolution, as proposed previously ${ }^{24}$. On the other hand, the high sequence conservation of both domain interfaces (Supplementary Fig. 1) strongly argues that the structures of ZIKV/JEV NS5 and DENV3 NS5 represent two alternative conformations of NS5 that may coexist in solution. Consistently, previous small-angle X-ray scattering analysis suggested the presence of a heterogeneous conformational ensemble of DENV3 NS5 in solution ${ }^{24}$, and mutations at the two alternative domain interfaces lead to compromised methyltransferase activity or viral replication function of DENV3 NS5 (ref. 19). Additional biochemical and cellular analyses are required to reveal the functional implication of these two alternative conformations of flavivirus NS5 proteins.

De novo RdRp assay of ZIKV NS5. To confirm that the ZIKV NS5 protein used for our structural study represents an active enzyme, we performed a de novo RdRp assay for ZIKV NS5 on a subgenomic ZIKV RNA template (Fig. 3a), using the recombinant ZIKV NS3 helicase domain (NS3-Hel) (Fig. 3b and Supplementary Fig. 4) as a negative control. We observed that the presence of ZIKV NS5 led to a time-dependent increase in the replication of the subgenomic ZIKV RNA at $33^{\circ} \mathrm{C}$ (Fig. $3 \mathrm{C}$ and Supplementary Fig. 5). However, the reaction product became dominated by a shorter RNA at $23^{\circ} \mathrm{C}$, possibly due to early termination of the replication (Fig. 3c). On the other hand, the presence of ZIKV NS3-Hel failed to yield any RNA product (Fig. 3c). Together, these data not only confirm that the ZIKV NS5 protein used for the structural study is enzymatically active but also provide a basis for further functional characterization of ZIKV NS5.

Identification of potential inhibitor-binding sites. Finally, we asked whether the structure of ZIKV NS5 permits us to identify potential inhibitor-binding sites for its enzymatic inhibition. A previous study, through fragment-based crystallography method, identified a pocket near to the active site of the DENV3 RdRp domain, termed ' $\mathrm{N}$ pocket', which binds to a smallmolecule that inhibits DENV3 NS5-mediated RNA initiation and elongation (Fig. 4a) ${ }^{25}$. Detailed analysis of this inhibitor-binding site revealed that the critical residues for the inhibitor binding are also conserved in ZIKV NS5, arranged in a similar structural environment (Fig. 4b); therefore, suggesting that the same compound may also be inhibitory to the enzymatic activity of ZIKV NS5. Further enzymatic analysis is needed to test the possibility of applying this DENV3 inhibitor to suppress the activity of ZIKV NS5.

\section{Discussion}

Our structural study of ZIKV NS5 sheds light onto the conformational dynamics and functional regulation of ZIKV NS5. The observation that ZIKV NS5 adopts one of the two defined conformations of flavivirus NS5 proteins provides first evidence on the conservation of domain conformation within NS5 proteins, which may be required for their functional regulation. To confirm the enzymatic activity of the recombinant ZIKV NS5 protein, we developed a de novo RdRp assay of ZIKV NS5 on a subgenomic ZIKV RNA template, which provides basis for future mechanistic characterization of ZIKV NS5. Furthermore, we identified that the small molecular inhibitorbinding site of DENV3 NS5 is structurally conserved in ZIKV NS5, thereby revealing a potential mechanism for functional inhibition of ZIKV NS5. This study provides a foundation for 
a

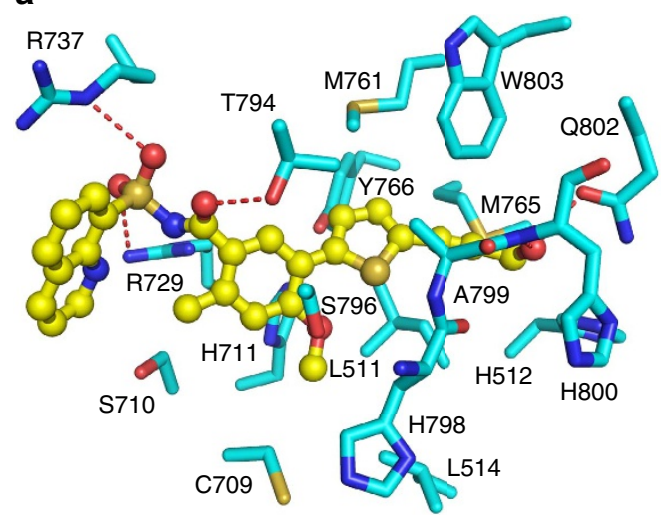

b

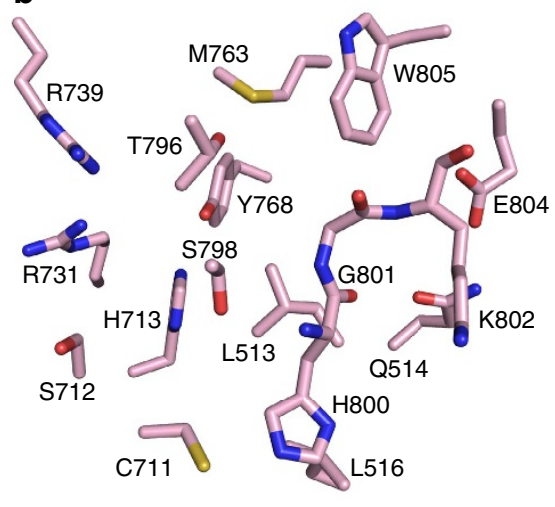

Figure 4 | Identification of a potential inhibitor-binding site in ZIKV NS5. (a) Binding of a small-molecule inhibitor of DENV3 NS5 at its 'N pocket'. The residues of DENV3 NS5 and small-molecule inhibitor are shown in blue and yellow sticks, respectively. The hydrogen bonding interactions are depicted as dashed lines. (b) The residues of ZIKV NS5 corresponding to the inhibitor binding site of DENV3 NS5 are shown in pink sticks.

future dissection of the functional coupling between the MTase and RdRp domains and a framework for the design of novel inhibitors against ZIKV infection.

\section{Methods}

Expression and purification of ZIKV NS5 and NS3 helicase. The DNA sequence encoding full-length ZIKV NS5 or ZIKV NS3-Hel (residues 171-617) was amplified from the cDNA of ZIKV/Macaca mulatta/UGA/MR-766/1947 and inserted into a modified pRSFDuet-1 vector (Novagen) (see Supplementary Table 1 for primer sequences), in which the NS5 or NS3-Hel gene was preceded by an N-terminal $\mathrm{His}_{6}$ SUMO tag and ULP1 (ubiquitin-like protease 1) cleavage site. The obtained plasmids were then transformed into BL21 (DE3) RIL cell strain (Agilent Technologies) for expression. The cells were first grown at $37^{\circ} \mathrm{C}$ and then shifted to room temperature when $A_{600}$ reached 1.0, followed by the addition of $0.4 \mathrm{mM}$ isopropyl $\beta$-D-galactoside for induction. After another $18 \mathrm{~h}$ of cell growth, the cells were collected and the $\mathrm{His}_{6}{ }_{-}$ SUMO-tagged ZIKV NS5 or ZIKV NS3-Hel was purified using a Ni-NTA affinity column. ZIKV NS5 was further purified on a Phenyl Sepharose column (GE Healthcare) for separation from degraded protein products, followed by removal of the $\mathrm{His}_{6}$-SUMO tag through ULP1 cleavage and size-exclusion chromatography on a Superdex 200 16/600 column (GE Healthcare) pre-equilibrated in buffer containing $25 \mathrm{mM}$ Tris, pH 7.5, $500 \mathrm{mM} \mathrm{NaCl}, 5 \mathrm{mM}$ DTT (dithiothreitol) and 5\% glycerol. The ZIKV NS3-Hel fusion protein was first subject to ULP1 cleavage, and subsequently purified on a Phenyl Sepherase column and a Superdex 200 16/600 column preequilibrated in buffer containing $25 \mathrm{mM}$ Tris, $\mathrm{pH} 7.5,250 \mathrm{mM} \mathrm{NaCl}, 5 \mathrm{mM}$ DTT and $5 \%$ glycerol. SDS-polyacrylamide gel electrophoresis analysis indicated that the purities of NS5 and NS3-Hel proteins were $>95 \%$ and $>90 \%$, respectively. Protein solution of purified NS5 and NS3-Hel, with concentrations of $\sim 20$ and $\sim 70 \mathrm{mg} \mathrm{ml}^{-1}$, respectively, were stored at $-80^{\circ} \mathrm{C}$.

Crystallization and X-ray data collection. Full-length ZIKV NS5 was mixed with SAH and GTP in a 1:3:3 molar ratio for complex formation. Initial crystallization conditions were identified through sparse-matrix screens (Hampton Research Inc.). The crystals were subsequently reproduced by hanging-drop vapour diffusion method at $4{ }^{\circ} \mathrm{C}$, from drops mixed from $1 \mu \mathrm{l}$ of ZIKV NS5 and $1 \mu \mathrm{l}$ of precipitant solution (0.7-0.9 M lithium sulfate, $0.1 \mathrm{M}$ MES, $\mathrm{pH}$ 6-7). Crystals were soaked for $1 \mathrm{~min}$ in a cryoprotectant solution, comprising of crystallization buffer and $20 \%$ glycerol, before flash frozen in liquid nitrogen.

The X-ray diffraction data for ZIKV NS5 were collected on the BL 5.0.3 beamline at the Advanced Light Source, Lawrence Berkeley National Laboratory. The diffraction data were indexed, integrated and scaled using the HKL2000 program ${ }^{26}$. The structure was solved using the molecular replacement method in PHASER $^{27}$, with the structure of Japanese encephalitis virus NS5 (PDB ID: 4K6M) as search model. The resulting electron density revealed that there are two molecules of ZIKV NS5 in each asymmetric unit. Despite being present in the crystallization mixture, GTP molecules were not modelled, presumably due to low occupancy under the crystallization condition. The structure of ZIKV NS5 was improved by iterative model building and refinement with $\operatorname{Coot}^{28}$ and PHENIX ${ }^{29}$ software packages. The same R-free test set was used throughout the refinement. The statistics for data collection and structural refinement of ZIKV NS5 is summarized in Table 1.

De novo RdRp assay. For ZIKV, the de novo RdRp reaction $(20 \mu \mathrm{l})$ contained $50 \mathrm{mM}$ Tris (pH 8.0), $10 \mathrm{mM} \mathrm{NaCl}, 5 \mathrm{mM} \mathrm{MgCl} 2,2 \mathrm{mM} \mathrm{MnCl}, 10 \mathrm{mM}$ DTT, $0.5 \mathrm{mM}$ ATP, $0.5 \mathrm{mM}$ UTP, $0.5 \mathrm{mM}$ GTP, $5 \mu \mathrm{M}$ CTP, $15 \mu \mathrm{Ci}$ of $\left[\alpha^{-32} \mathrm{P}\right]$

\begin{tabular}{|c|c|}
\hline & ZIKV NS5 \\
\hline \multicolumn{2}{|l|}{ Data collection } \\
\hline Space group & $P 22_{1} 2_{1}$ \\
\hline \multicolumn{2}{|l|}{ Cell dimensions } \\
\hline$a, b, c(\AA)$ & $95.1,136.5,197.0$ \\
\hline$\alpha, \beta, \gamma\left(^{\circ}\right)$ & $90,90,90$ \\
\hline Wavelength & 0.9764 \\
\hline Resolution $(\AA)$ & $50.00-3.30(3.42-3.30)^{\star}$ \\
\hline$R_{\text {sym }}$ or $R_{\text {merge }}$ & $31.3(83.5)$ \\
\hline$|/ \sigma|$ & $5.3(1.7)$ \\
\hline Completeness (\%) & $99.4(97.1)$ \\
\hline Redundancy & $6.2(5.7)$ \\
\hline $\mathrm{CC}_{1 / 2}$ & $0.979(0.748)$ \\
\hline \multicolumn{2}{|l|}{ Refinement } \\
\hline Resolution $(\AA)$ & $48.3-3.28(3.36-3.28)$ \\
\hline No. of reflections & $39,405(3,511)$ \\
\hline$R_{\text {work }} / R_{\text {free }}$ & $0.262 / 0.293(0.344 / 0.405)$ \\
\hline \multicolumn{2}{|l|}{ No. of atoms } \\
\hline Protein & 13,391 \\
\hline Ligand & 38 \\
\hline \multicolumn{2}{|l|}{$B$ factors } \\
\hline Protein & 54.12 \\
\hline Ligand & 68.85 \\
\hline \multicolumn{2}{|l|}{ r.m.s.d. } \\
\hline Bond lengths $(\AA)$ & 0.002 \\
\hline Bond angles $\left({ }^{\circ}\right)$ & 0.55 \\
\hline
\end{tabular}

*Values within parentheses are for highest-resolution shell.

( $10 \mu \mathrm{Ci}_{\mu \mathrm{l}^{-1}}^{-1}, 3,000 \mathrm{Ci} \mathrm{mmol}^{-1}$; Perkin-Elmer), $1 \mu \mathrm{g}$ of RNA template and $2 \mu \mathrm{g}$ of ZIKV NS5 protein or ZIKV NS3-Hel. The RNA template was in vitro transcribed from a PCR product using T7 polymerase (New England BioLabs). The PCR product contained a T7 promoter, followed by a cDNA fragment representing a ZIKV subgenome with deletion of nucleotides 171-10,343 (GenBank accession no. KU963573.2). The de novo RdRp reaction mixtures were incubated at $23^{\circ} \mathrm{C}$ for $30 \mathrm{~min}$, or $33^{\circ} \mathrm{C}$ for 30,60 and $120 \mathrm{~min}$. The final reactions were further extracted with phenol-chloroform and precipitated with isopropanol. The RNA pellet was dissolved in $20 \mu \mathrm{l}$ of $1 \times$ denaturing gel loading dye, and loaded onto a $10 \%$ denaturing polyacrylamide gel with $7 \mathrm{M}$ urea. ${ }^{32} \mathrm{P}$-labelled RNA results were detected via the autoradiograph of the PAGE gel.

Data availability. Coordinates and structure factors for ZIKV NS5-SAH complexes have been deposited in the Protein Data Bank under accession code 5TMH. The PDB accession codes 5TMH, 5JJR, 4K6M and 4V0Q and GenBank entry KU963573.2 were used in this study. All other data are available from the corresponding authors on reasonable request. 


\section{References}

1. Duffy, M. R. et al. Zika virus outbreak on Yap Island, Federated States of Micronesia. N. Engl. J. Med. 360, 2536-2543 (2009).

2. Faria, N. R. et al. Zika virus in the Americas: early epidemiological and genetic findings. Science 352, 345-349 (2016).

3. Rasmussen, S. A., Jamieson, D. J., Honein, M. A. \& Petersen, L. R. Zika virus and birth defects-reviewing the evidence for causality. N. Engl. J. Med. 374, 1981-1987 (2016).

4. Cao-Lormeau, V. M. et al. Guillain-Barre Syndrome outbreak associated with Zika virus infection in French Polynesia: a case-control study. Lancet 387, 1531-1539 (2016).

5. Petersen, L. R., Jamieson, D. J., Powers, A. M. \& Honein, M. A. Zika virus. N. Engl. J. Med. 374, 1552-1563 (2016).

6. Shan, C. et al. An infectious cDNA clone of Zika virus to study viral virulence, mosquito transmission, and antiviral inhibitors. Cell Host Microbe 19, 891-900 (2016).

7. Potisopon, S. et al. The methyltransferase domain of dengue virus protein NS5 ensures efficient RNA synthesis initiation and elongation by the polymerase domain. Nucleic Acids Res. 42, 11642-11656 (2014).

8. Ashour, J. et al. Mouse STAT2 restricts early dengue virus replication. Cell Host Microbe 8, 410-421 (2010).

9. Best, S. M. et al. Inhibition of interferon-stimulated JAK-STAT signaling by a tick-borne flavivirus and identification of NS5 as an interferon antagonist. J. Virol. 79, 12828-12839 (2005).

10. Grant, A. et al. Zika virus targets human STAT2 to inhibit type I interferon signaling. Cell Host Microbe 19, 882-890 (2016).

11. Laurent-Rolle, M. et al. The NS5 protein of the virulent West Nile virus NY99 strain is a potent antagonist of type I interferon-mediated JAK-STAT signaling. J. Virol. 84, 3503-3515 (2010).

12. Laurent-Rolle, M. et al. The interferon signaling antagonist function of yellow fever virus NS5 protein is activated by type I interferon. Cell Host Microbe 16, 314-327 (2014).

13. Lin, R. J., Chang, B. L., Yu, H. P., Liao, C. L. \& Lin, Y. L. Blocking of interferon-induced Jak-Stat signaling by Japanese encephalitis virus NS5 through a protein tyrosine phosphatase-mediated mechanism. J. Virol. 80, 5908-5918 (2006)

14. Lubick, K. J. et al. Flavivirus antagonism of type I interferon signaling reveals prolidase as a regulator of IFNAR1 surface expression. Cell Host Microbe 18, 61-74 (2015).

15. Mazzon, M., Jones, M., Davidson, A., Chain, B. \& Jacobs, M. Dengue virus NS5 inhibits interferon-alpha signaling by blocking signal transducer and activator of transcription 2 phosphorylation. J. Infect. Dis. 200, 1261-1270 (2009).

16. Morrison, J. et al. Dengue virus co-opts UBR4 to degrade STAT2 and antagonize type I interferon signaling. PLoS Pathogens 9, e1003265 (2013).

17. Lim, S. P., Noble, C. G. \& Shi, P. Y. The dengue virus NS5 protein as a target for drug discovery. Antiviral Res. 119, 57-67 (2015).

18. Lu, G. \& Gong, P. Crystal structure of the full-length Japanese encephalitis virus NS5 reveals a conserved methyltransferase-polymerase interface. PLoS Pathogens 9, e1003549 (2013).

19. Zhao, Y. et al. A crystal structure of the dengue virus NS5 protein reveals a novel inter-domain interface essential for protein flexibility and virus replication. PLoS Pathogens 11, e1004682 (2015).

20. Ferrer-Orta, C., Arias, A., Escarmis, C. \& Verdaguer, N. A comparison of viral RNA-dependent RNA polymerases. Curr. Opin. Struct. Biol. 16, 27-34 (2006).

21. Coloma, J., Jain, R., Rajashankar, K. R., Garcia-Sastre, A. \& Aggarwal, A. K. Structures of NS5 methyltransferase from Zika virus. Cell Rep. 16, 3097-3102 (2016).

22. Iglesias, N. G., Filomatori, C. V. \& Gamarnik, A. V. The F1 motif of dengue virus polymerase NS5 is involved in promoter-dependent RNA synthesis. J. Virol. 85, 5745-5756 (2011).
23. Yap, T. L. et al. Crystal structure of the dengue virus RNA-dependent RNA polymerase catalytic domain at 1.85 -angstrom resolution. J. Virol. 81, 4753-4765 (2007).

24. Bussetta, C. \& Choi, K. H. Dengue virus nonstructural protein 5 adopts multiple conformations in solution. Biochemistry 51, 5921-5931 (2012).

25. Lim, S. P. et al. Potent allosteric dengue virus NS5 polymerase inhibitors: mechanism of action and resistance profiling. PLoS Pathogens 12, e1005737 (2016).

26. Otwinowski, Z. \& Minor, W. Processing of X-ray diffraction data collected in oscillation mode. Method Enzymol. 276, 307-326 (1997).

27. McCoy, A. J. et al. Phaser crystallographic software. J. Appl. Crystallogr. 40, 658-674 (2007).

28. Emsley, P. \& Cowtan, K. Coot: model-building tools for molecular graphics. Acta Crystallogr. D 60, 2126-2132 (2004).

29. Adams, P. D. et al. PHENIX: building new software for automated crystallographic structure determination. Acta Crystallogr. D 58, 1948-1954 (2002).

\section{Acknowledgements}

This work was supported by March of Dimes Foundation (1-FY15-345), Kimmel Scholar Award from Sidney Kimmel Foundation for Cancer Research and NIH (1R35GM119721) (to J.S.). The Berkeley Center for Structural Biology is supported, in part, by the National Institutes of Health, National Institute of General Medical Sciences and the Howard Hughes Medical Institute. The Advanced Light Source is supported by the Director, Office of Science, Office of Basic Energy Sciences and of the US Department of Energy under Contract No DE-AC02-05CH11231.

\section{Author contributions}

B.W., S.T. and R.H. cloned ZIKV NS5. B.W., A.L. and J.S. purified the protein sample. B.W. and X.-F.T. crystallized ZIKV NS5 and collected the X-ray data set. X.-F.T. and Z.-M.Z. determined the crystal structure. R.H. and S.T. performed enzymatic assay. R.H. and J.S. organized the study. J.S. prepared the manuscript with input from all the authors.

\section{Additional information}

Supplementary Information accompanies this paper at http://www.nature.com/ naturecommunications

Competing financial interests: The authors declare no competing financial interests.

Reprints and permission information is available online at http://npg.nature.com/ reprintsandpermissions/

How to cite this article: Wang, B. et al. The structure of Zika virus NS5 reveals a conserved domain conformation. Nat. Commun. 8, 14763 doi: 10.1038/ncomms14763 (2017).

Publisher's note: Springer Nature remains neutral with regard to jurisdictional claims in published maps and institutional affiliations.

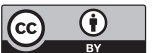

This work is licensed under a Creative Commons Attribution 4.0 International License. The images or other third party material in this article are included in the article's Creative Commons license, unless indicated otherwise in the credit line; if the material is not included under the Creative Commons license, users will need to obtain permission from the license holder to reproduce the material To view a copy of this license, visit http://creativecommons.org/licenses/by/4.0/

C) The Author(s) 2017 